\title{
Peepdeck: a dashboard for the distributed design studio
}

\author{
Jesús Muñoz-Alcántara \\ Eindhoven University of \\ Technology \\ De Rondom 70, 5612AP, \\ Eindhoven, The Netherlands \\ Email: j.munoz.alcantara@tue.nl
}

\author{
Petr Kosnar, \\ Eindhoven University of \\ Technology \\ De Rondom 70, 5612AP, \\ Eindhoven, The Netherlands \\ Email: hello@iampetr.com
}

\author{
Mathias Funk, Panos \\ Markopoulos, \\ Eindhoven University of \\ Technology \\ De Rondom 70, 5612AP, \\ Eindhoven, The Netherlands \\ Email: \{m.funk, p.markopoulos \\ @tue.nl\}
}

\begin{abstract}
Designers adopt a large amount of generalpurpose tools for supporting their remote collaborative tasks. Each tool provides very diverse functionalities: from file sharing to instant communication and video collaboration. The designer struggles when filtering and combining the right information spread across the multitude of tools. This research extends McGrath's framework of task circumflex to map the collaborative demands of the design practitioner and proposes Peepdeck, a design exploration to support them. Peepdeck is a dashboard that assembles information scattered across multiple tools in a personalized and organized way. Through two design iterations followed by evaluations of the user interface, several requirements were identified for supporting collaboration awareness in design teams. Insights confirmed the relevance of combining information from different but already familiar tools, rather than attempting to replace them. It was identified the importance of optimizing for visual scanning, supporting search of content and allowing users to customize the tool.
\end{abstract}

\section{INTRODUCTION}

$\mathrm{D}$ esign studios operate in a much more distributed fashion nowadays than in the past. Designers may be distributed geographically, potentially even in different time zones. This paper discusses the design of an application for supporting distributed collaborative design work. There is a myriad of new tools and online services available as shown by Fig 1. Tools like Dropbox, GoogleApps, Basecamp, Atlassian, Slack, Skype and Trello are just some examples of the diversity of commercial applications available on the Internet that support teamwork in the design studio. These technologies support very diverse functionalities and services, from file sharing, online edition of documents to instant communication and video collaboration.

Previous research [13] revealed a collection of patterns of behavior that the designers conduct in the context of collaboration tools. A surprising finding was that designers adopt a large amount of generic tools that they appropriate for supporting collaborative design tasks, choosing different tools for different parts of the design process. Tools like

This work was funded by the COnCEPT project as part of the European Commission 7th Framework ICT Research Programme: Project Number 610725. For further details visit: http://www.concept-fp7.eu
Facebook (social media), Dropbox (file sharing) and Skype (instant communication) were some of the most popular.

While some of the tools are used throughout the whole design process, each of the categories of behavior imposes its own challenges to each of the tools. Individuals switch fluently from one tool to another depending on the activity, the personal needs, the project needs and how the tool covers those needs. The designer must be able to filter and combine the right information spread across the given multitude of tools, each with different information (file, contact, folder, application) and user interfaces. This is the starting point that motivates the present work in order to facilitate the current collaborative practices of designers.

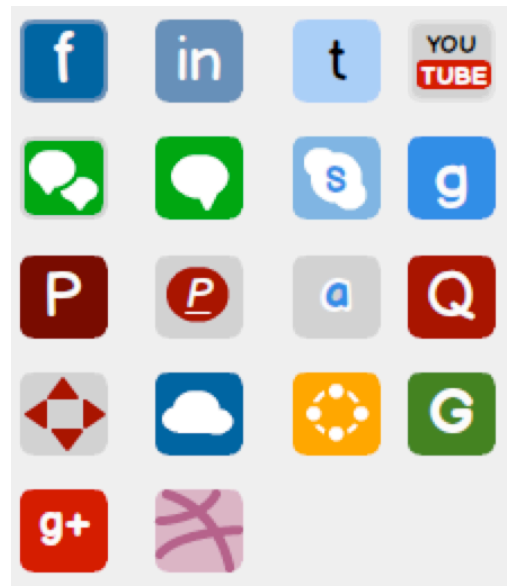

Fig 1. Set of icons representing the diversity of commercial applications available on the Internet. Original icons by Designyantra used under Creative Commons License.

The current research presents a design exploration into how collaboration practices for distributed design teams can be supported. The paper starts by taking as reference McGrath's framework of task circumflex [12] to analyze how the current collaboration tools are incapable of covering the demands of the design practitioner. Then, the paper proposes as a solution an application called Peepdeck, which assemble information scattered across multiple tools in a personalized and organized way. Afterwards, the two iterations of the design and evaluation of the user interface of the application are presented. Finally, the paper discusses a reflection to the proposed design, the design requirements 
and further issues pertaining the design of such groupware applications.

\section{Fitting TeChNOLOGY IN PRACTICE}

The vast amount of existing research in the field of CSCW [21] has illustrated how groups adopted IT tools and integrated them into their social dynamics to support teamwork. Researchers have identified specific patterns of behavior supported by the appropriation of collaboration tools $[10 ; 17 ; 18 ; 19]$ and how those patterns changed through their appropriation [24].

McGrath [12] defined a framework, called task circumflex, to classify technology according to the type of task that they were supporting within the team. McGrath classified collaboration tools in 4 quadrants based on whether they supported (1) generation activities: for planning and idea creation, (2) choice activities: for problem solving and decision-making, (3) execution activities: for the task execution and performance, or (4) negotiation activities: for tasks that focus in resolving conflicts between the individuals (Fig 2).

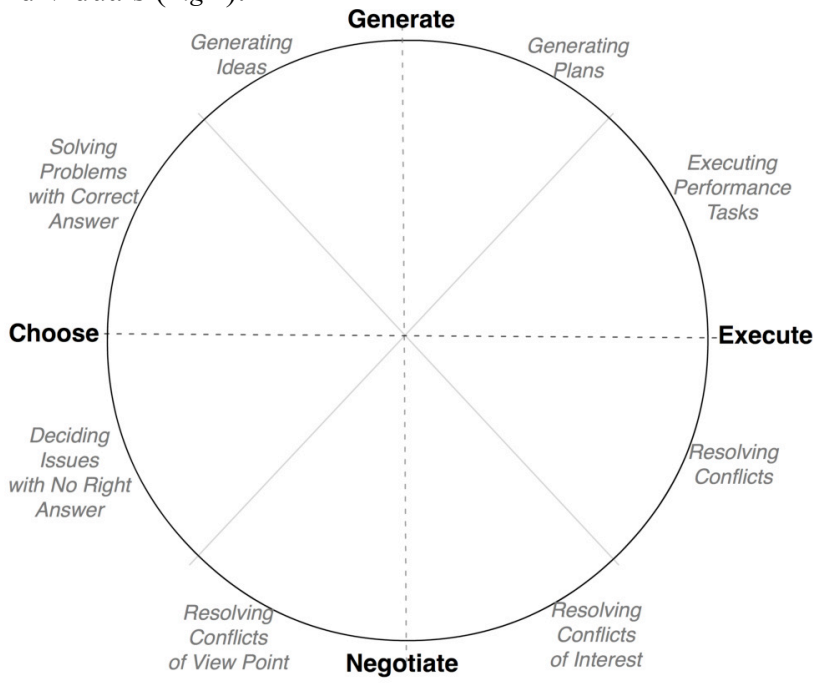

Fig 2. The task circumflex defined by McGrath (1984)

In the context of remote design teams, Muñoz-Alcántara and colleagues [13] performed a series of interviews and surveys to professional designers in order to understand the activities that designers engage to support their collaborative practices. Their results described 5 categories of collaborative activities: (1) creating ideas and concepts (e.g., ideation, brainstorming and inspiration), (2) developing ideas and concepts (e.g., sketching and prototyping), (3) making sense of the material, resources, and experiential knowledge (e.g., giving and receiving feedback), (4) keeping the team on track (e.g., notifying the team and solving issues in the team), and (5) managing the development of the project (e.g., defining and managing tasks and deadlines). As shown in Fig 3, the first 3 categories described the core activities of the design practice, while the last 2 , described the social dynamics that enable the completion of the first set of processes.

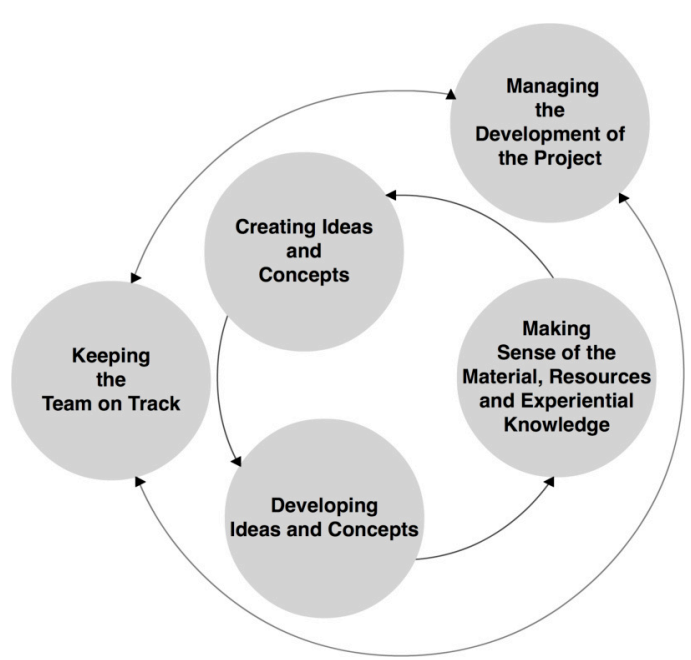

Fig 3. This diagram shows the flow of the five categories of collaborative activities described by Muñoz-Alcántara et al. (2015)

Additionally, Muñoz-Alcántara et al. [13] compiled a list of specific tools that designers use during each of the activities, as shown in Table I. The list reveals that professional designers use few specialized tools compared to the total amount of tools involved during the creative stages of their work. Furthermore, Table I illustrates how various tools appear repeatedly in more than one of the activities. This clearly shows that a large collection of different tools supports the designers on more than one of their daily tasks.

TABLE I. LIST OF TOOLS USED ON EACH DESIGN ACTIVITY

\begin{tabular}{c|c|}
\hline Activity & \multicolumn{1}{|c|}{ Tools involved } \\
\hline $\begin{array}{c}\text { Creating ideas } \\
\text { and concepts }\end{array}$ & $\begin{array}{c}\text { Google Docs, Google Spreadsheets, } \\
\text { Mindmaps, Skype, Evernote, Dropbox, } \\
\text { Pinterest, Facebook }\end{array}$ \\
\hline $\begin{array}{c}\text { Developing ideas } \\
\text { and concepts }\end{array}$ & $\begin{array}{l}\text { Paper, iPad (Adobe Ideas, Paper 53), } \\
\text { Axure, Excel, Illustrator, SVN, Github, } \\
\text { Bitbucket, Dropbox, Google Docs }\end{array}$ \\
\hline $\begin{array}{c}\text { Making sense of } \\
\text { the material, etc. }\end{array}$ & $\begin{array}{l}\text { Skype, Evernote, Facebook Groups, } \\
\text { Facebook Chat, Lynk, email and Google } \\
\text { Hangouts, Email, WeTransfer, Dropbox, } \\
\text { Axure, Interactive documents }\end{array}$ \\
\hline $\begin{array}{c}\text { Keeping the team } \\
\text { on track }\end{array}$ & $\begin{array}{l}\text { Facebook Group, Facebook Chat, } \\
\text { Skype, email, Whatsapp }\end{array}$ \\
\hline $\begin{array}{c}\text { Medmine, Gantt charts, Teambox, } \\
\text { development of the } \\
\text { project }\end{array}$ & $\begin{array}{l}\text { spreadsheets, Trello, Outlook, Google } \\
\text { Docs, Dropbox, Google Drive, Facebook } \\
\text { Groups }\end{array}$
\end{tabular}

Each of these collaborative processes can be mapped into the classification given by McGrath in order to reflect how each tool is used on every stage of the creative design process. The core process of creating ideas and concepts belongs to the quadrants of generation support tools and choice support tools. Tools aimed for generation and for execution activities mainly support the process of developing ideas and concepts. The process of making sense of the material is mainly covered by the quadrant of tools for choice but it also includes tools focused on negotiation. Keeping the team on track is primarily achieved by the support of negotiation tools while managing the development of the project depends on generation and 
execution means. Fig 4 displays the task classification defined by McGrath combined with the group activities described by Muñoz-Alcántara and colleagues. These sets of behaviors provide the starting point for understanding how each different activity provides a specific value to the effort of the design team.

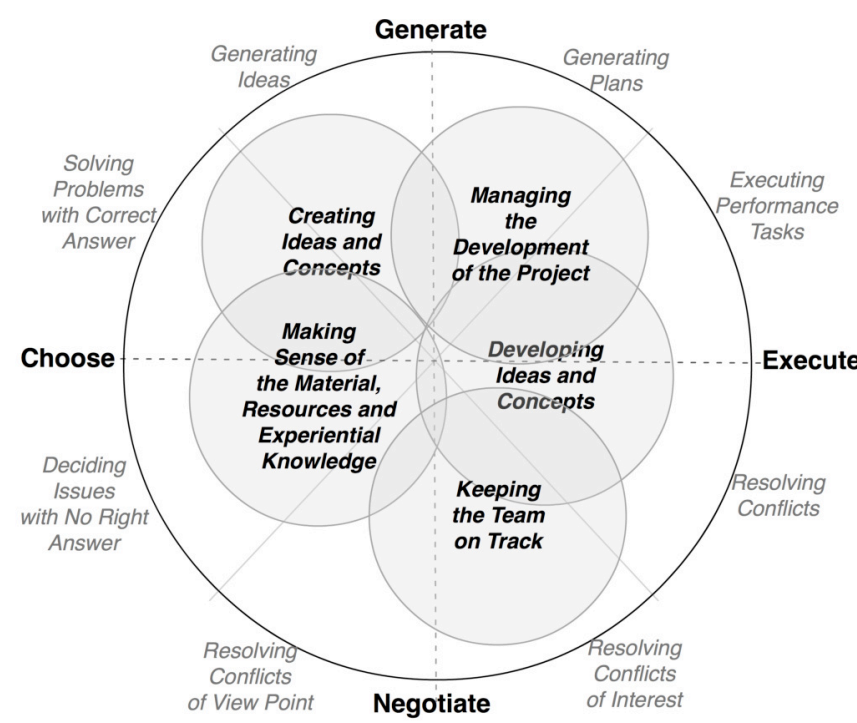

Fig 4. This diagram maps the task circumflex of McGrath (1984) with the specific patterns of behavior of a designer described by MuñozAlcántara et al. (2015).

Traditionally, design studios have a high material character. For example, office walls and desks are typically full of notes, post-it, sketches, prototypes and physical models. The material and physical aspects embedded in the design studio such as designer's practices, the use of artifacts and the space during collaboration, have a key role on the coordination of the creative activities [23]. Design artifacts (e.g. sketches, physical prototypes) located in the studio inform activity and progress of the group and trigger awareness in the team [2,6]. The use of space is also productivity-focused, displaying information that supports time management, scheduling and division of workload. The designer takes advantage of the space and the artifacts to enable the coordination with the team. The design process often demands fast switching between activities, and thus, between artifacts and tools. Most activities require the selection and combination of information spread across several sources. Moreover, every person requires a unique set of information based on the roles, responsibilities, and also personal preferences and interest in the projects they are working on.

However, on a distributed setting, the digital information is usually managed and visualized through very different interfaces and organized in different information architecture. Since general-purpose tools are generally adopted to support certain design activities, extra work is needed for annotations, documentation, and organizing files and folders on each of the tools. As a consequence designers fail to integrate and coordinate the information, effort and outcomes of other team member's activities. Furthermore, when using a large collection of tools it is difficult to have an overview of the design process and the performance of the individual is affected by the huge amount of information provided by the collection of tools.

The question raised is how a groupware application could support design collaboration by focusing on the integration of the current tools while addressing the core requirements of the design tasks.

The design of the user interface of the dashboard followed a user-centered approach with two iterative cycles. Each design iteration consisted of the following steps: specification finding, conceptualization, development of the concept through prototyping and the evaluation of the prototype. The first iteration focused on creating and evaluating the concept of the dashboard. Drawing from the problems we identified during the literature research, this iteration explored what is the meaningful content of such a tool, what is the way how designers can use it, its information architecture and how they can incorporate it into their typical work routine. This was first evaluated on a paper prototype. In the second iteration an interactive digital prototype was developed and its UI was evaluated in terms of interactions, ease of use, and clarity of the application. The final outcome of this work is to bridge the gap between the existing qualitative fieldwork studies and the design recommendations based on the actual design and evaluation of a tool that enables remote collaboration in the design practice.

\section{INITIAL CONCEPT}

The insights gathered from the literature review suggested to focus on solving the problem of scattered information and losing oversight rather than creating yet another specialized tool that solves narrowly scoped problems for designers. The concept of a dashboard emerged. The dashboard aggregates information from (multiple) existing tools and services, and displays them in a personalized manner at one place. This approach enables keeping a single UI for the user regardless of the actual tools and data sources integrated on a lower level. As one of the consequences, exchanging of the backend infrastructure or the connected tools does not need to be noticeable by the users.

\section{FIRST DESIGN ITERATION}

\section{Design specifications}

The success of the dashboard mainly depends on addressing the challenge of accessibility of the information and implementation complexity. A number of design challenges were identified on which the success of the dashboard depends:

- Making a glanceable display [11] that collects salient information for design collaboration in one place?

- What is the right amount of information? [4] (Lack of awareness vs. Information overload) 
- How to design simple and intuitive interface?

(Minimizing learning curve $[15 ; 16]$ )

- Information visualisation: overview / information display

(Optimized for visual searching [8;9])

The dashboard should incorporate the existing tools and provide an extra layer where designers can access the selected content and information streams, and filter them as (individually) preferred. Finally, it should also enable users to stay updated by checking only a single place (tool), instead of multiple different tools and their different notification areas, status bars, streams, feeds, and other elements meant for updating the user.

\section{Peepdeck concept}

The Peepdeck concept was conceived to address the challenges identified above. Peepdeck is envisioned as an online service that connects existing tools and services that users already use for collaborative design; it aggregates information from the connected services, and classifies it into four main categories resolved from the common groups of items described in the enabling activities discussed above: tasks management, shared calendar items, communication streams, and cloud storage with file management facilities. These categories contain information merged from various sources in a way that the information does not define the attributes or features of the item (i.e., a file from Dropbox is treated equally as a file from OneDrive, or Google Drive).

The central goal of Peepdeck is to display the information that is currently relevant to the user - reflecting their role, context, projects they are involved in, co-workers, personal preferences, and other aspects. The user can adjust the content of the dashboard so it matches his requirements. The dashboard contains shortcut to the connected services - so the user does not replace them with the Peepdeck, but
Peepdeck just enables easy and quick access to the important parts (folders, files, and other items) in each connected tool.

\section{Concept development}

Concept development was the goal of the first iteration. After defining the concept of the Peepdeck as described above we created minimal version of the UI in a form of a wireframe, and then created a paper prototype that we used in the following user research.

\section{Wireframe}

The concept of the UI consisted of the vertical columns placed one next to each other (see Fig 5). Each of these columns consists of a title and number of items of different types. These items could be in a stream of information from selected source - such as stream of the updates of files from the connected cloud storage, or stream of the activities of the teammates.

The content of each item in each stream is dependent on the nature of the item, which can be a file, an activity, a calendar item, or a task. The items that are not in a stream represent a list of tasks, list of persons, or a block of multiple items of a different character. Variants of the prototype represented different levels of complexity for different items.

A wireframe was designed that was aimed at minimizing the content, abstracting away from the source of the content, and focusing on the type of information. That means, that the items of the same character (e.g., file, task) are represented on the screen similarly, regardless of the source of the item (e.g., whether the file is from Dropbox, OneDrive, or Google Drive). This way, the items are displayed with emphasis on their meaning, instead of structural features such as location where they are stored.
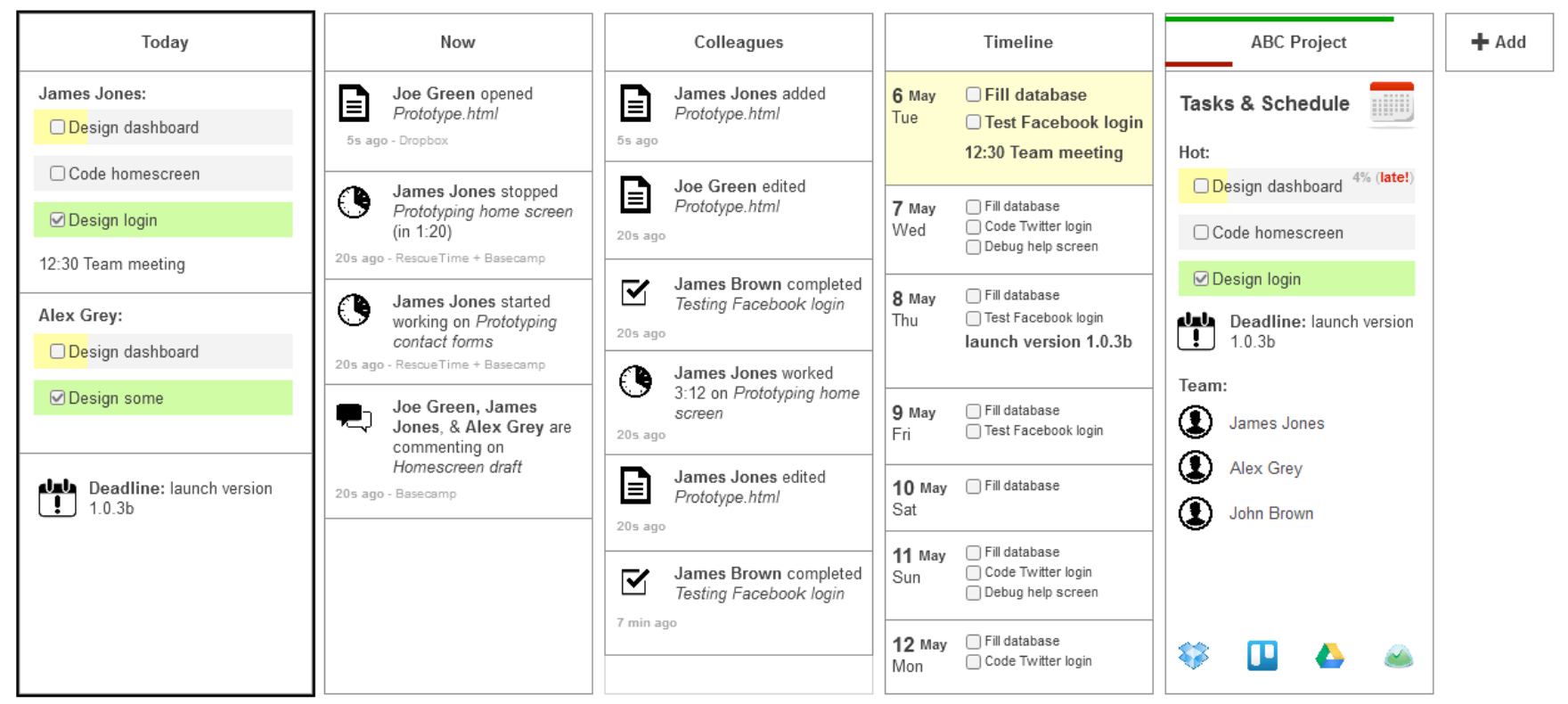


\section{Evaluation}

The purpose of the evaluation was to validate the design concept and to investigate how to display relevant content at a single place. The test sessions also explored the amount and type of information and how to combine the information in a simple and intuitive way.

A formative user test was conducted with seven participants (six males and one female, with an average age of 30 years old). All participants were full-time employed designers working in the Netherlands on various design positions such as interaction designer, industrial designer, usability engineer, game designer, or graphic designer. All of them worked at middle- to large-size Dutch or international companies, in a team of six to ten people, some of them in multiple teams at the same time. User sessions were conducted in English; the interviewer and the five participants were not native English speakers, however all were proficient English speakers and no communication difficulties were noticed. Each session took about 60 minutes.

The user test involved a combination of a semi-structured interview and thinking-aloud method followed by co-design activities, where participants proposed alternative designs. . In the beginning of the session the interviewer asked about tools currently used, the size of the team, the roles of their current teammates, and typical information required in their work. Additionally, they were asked about the way they collaborate, and what tools or methods they use in their team.

For the evaluation, a paper prototype of the interface was created from the wireframe models so that interactions could be simulated by manipulating paper cut outs (Fig 6). We experimented with excluding different types of items to test whether users actually miss them. Participants were given a set of tasks pertaining to understanding one's current state in the context of the status of tasks, projects, deadlines, files and the activities of other team member activities. During the tasks participants were thinking aloud, and after the (successful or unsuccessful) completion of each task, the interviewer asked about the relevance of the elements of the dashboard for completing that task.

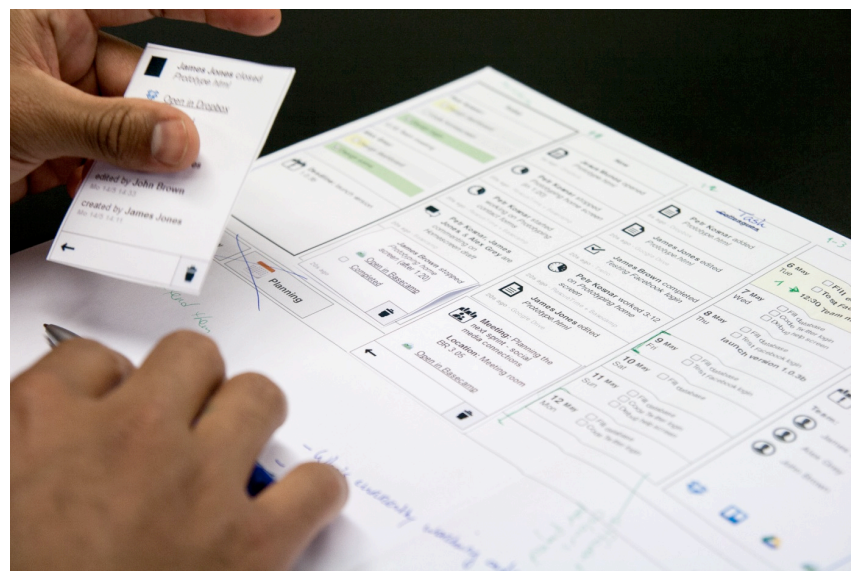

During the whole session both participant and interviewer had pens and markers available (each of different color) and participant was asked to add information into the dashboard that was missing for completing any task. Participants were also asked to cross out information that they find useless, or draw extra elements of the dashboard they are missing (Fig 6).

\section{Results}

The results of the user test revealed that the concept of the dashboard was evaluated very positively. Participants often related the UI elements, interaction or their expectations about the interface to the existing tools they were familiar with; and not only tools they use for work. Examples of the UI elements they mentioned were "badge counters" known from iOS applications, showing in a small field at the corner of the app's icon number of notifications this app has; stacking information as known from Facebook (e.g., when an item is shared by multiple persons, the item is shown once with the list of all persons who shared it, instead of repeating the item for each person); or iconography known from OS X and Facebook. In general, participants often related to the tools they were using at work already, which confirmed previous findings about the list tools they actually use. Participants usually asked for better visual clarity, showing context of the items and relationships between related items. All participants executed the tasks in different ways suggesting the need to allow flexibility in the user interface.

In terms of general problems and requirements, participants stated that they often work on multiple projects at the same time, or switch between projects every few days during a month. Therefore, the interface should support multiple project views and easy handling of them (adding, hiding, showing, and removing). The presented categories of items (such as events, tasks, files and messages) were understood, however the relationships between items on the dashboard and the persons or projects were not clear. Especially for project-related items such as tasks or deadlines, they missed clear hints about the project it relates to. The opinions and preferences on how to present extra information were divergent. A common remark was that the dashboard must be easy to scan visually supported by colors, spacing and the typography.

When referring to missing information or features, the most common concern was to include filtering and search functions. Participants referred to the possibility to stack similar items together, manipulate content (list of tasks, current files), edit privacy settings (shared or not shared), and to show and hide details of each item (tasks' status, deadlines, last time of synchronization). Besides, each of the items should have a link to the original source and other related items. The dashboard should also include the availability of colleagues (chat, IM, call, personal meeting). An integrated social media stream was also suggested with the possibility to disable specific applications when they do not want to be disturbed.

Fig 6. User testing of the version 1 of Peepdeck on a paper prototype 


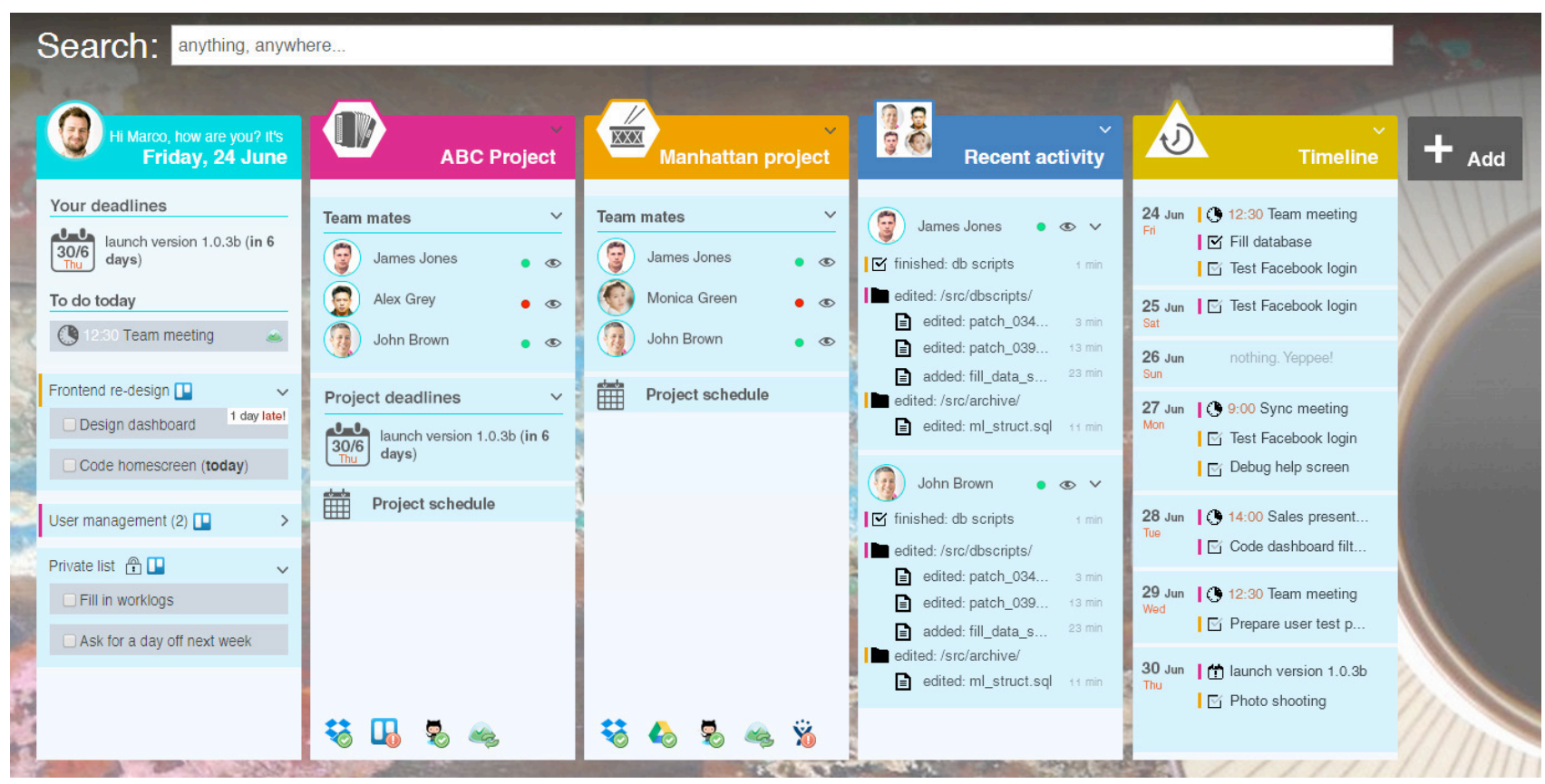

Fig 7. Final version of the UI of Peepdeck on a high-fidelity prototype

\section{V.SECOND DESIGN ITERATION}

The second iteration focused on creating and testing an interactive digital prototype implementing the guidelines resulting from the first iteration and user test. For instance, visual clarity, spaciousness and segmentation of the interface should dominate in order to support visual searching and effective scanning of the updates on the dashboard. Other components such as integrated search and availability of team members were included in the UI.

\section{UI design}

The wireframe of the first version of the dashboard was redesigned and then with the help of a mood board a visual design was created using colors and shapes with a meaning to support visual searching and peripheral scanning of the dashboard [1]. The interface, shown in Fig 7, consists of several columns, where the basic set of columns included: a "Today" column, a "Project" column, a "Team activity" column and a "Timeline" column. A full text search is placed visibly above all, to indicate that it searches all the content of the dashboard.

The "Today" column contains today's date and day of the week, photo and greeting of the user to attract attention and make user start reading the screen here. Then it lists the upcoming deadlines, lists of tasks, and a private list of tasks that are stored locally, and not shared. The "Project" column contains the header with the project name and an icon. This column contains a list of teammates, which are aggregated from tools that contain the defined team (e.g., Basecamp), or the users from the shared project folder (e.g., Dropbox). Each person has an indicator of availability on any connected communication service, and a visibility icon that can toggle the content related to this person in the whole dashboard. Underneath there is a list of upcoming deadlines of this project, a link to the project schedule (in selected tool where the project shares the calendar), and icons of all connected tools/services with the indication of synchronization status; these icons are shortcuts to the relevant project pages (e.g., Dropbox folder, or Basecamp project). The "Team activity" column contains the blocks of users and their recent activities such as edited files, or completed tasks. The "Timeline" column lists the simple linear calendar view with the upcoming events such as deadlines, meetings, and tasks. Users can add more columns into the dashboard, which will scroll horizontally.

\section{Prototype development}

An interactive prototype with high visual refinement based was created in the software Axure RP Pro 7.0. The result was an interactive mockup on HTML, CSS and JavaScript. The prototype implemented the designs created in the previous stage. It also added interactions allowing to manipulate the interface, such as collapsing and expanding the elements in the columns, hiding and adding new columns, do full text search with autocomplete function showing the search results during typing of the search phrase, adding new items on the private task list, hiding and showing content of each person on the dashboard, and showing extra information about elements on mouse hover. The prototype displayed in Fig 7 is the improved version after the insights from the second evaluation. The prototype is available on the URL: http://ojwzlv.axshare.com/.

\section{III.Evaluation}

User tests were conducted with seven designers (four males and three females, all in their early 30s). All participants were employed full-time at the time of the tests by middleto large-size Dutch or international companies, and they work in a team of six to ten people, often being part of multiple teams at the same time. The individuals held various design positions such as interaction designer, 
industrial designer, usability engineer, game designer, or graphic designer. User sessions were conducted in English. Each session took about 60 minutes.

The goal of the evaluation was to test the UI, regarding its visual clarity, the information architecture and the amount of information presented. The connections and data on the dashboard were simulated. User interactions with the interface were specially observed: how they explore and understand the elements on the screen. The terminology and wording used in the interface were also evaluated.

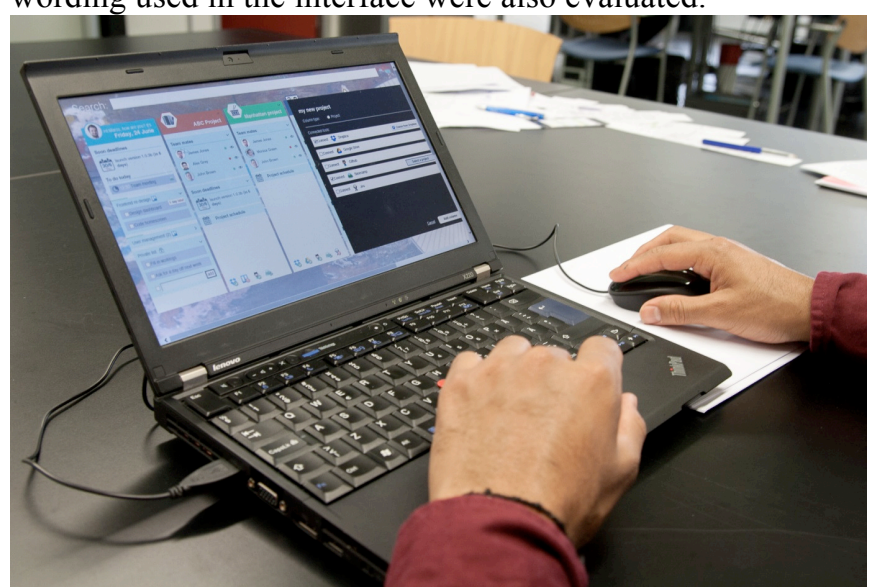

Fig 8. User evaluation of the version 2 of Peepdeck's UI using an the interactive prototype

The interactive prototype was tested on a computer with a mouse, in a full screen mode (Fig 8). A similar protocol to the first evaluation was executed during this evaluation. The user test was set up as a combination of a semi-structured interview, thinking-aloud while completing given tasks, and observation. The prototype was presented to participants, and then they were asked to complete a set of tasks while thinking aloud. These tasks were very similar to set of task performed during the first evaluation. The tasks pertained to the current status of one's activities, other member's activities, deadlines and projects. Some tasks focused on the added features such searching for a particular file, directly contacting a team member or unsubscribing from certain notifications. After each attempt the interviewer was asked about which elements of the dashboard were helpful for completing this task, and which parts of the dashboard were not. At the end, participants were asked several open-ended questions about situations in which they find this tool useful, and in which they think it would not be useful.

\section{Results}

In summary, the feedback on the tool was very positive as some participants noted that they wanted or needed such a tool already. The visual segmentation and organization of the dashboard was positively evaluated, and people reported that searching was easy, and clear. The results showed that users want to customize the order of the sections - natural action is drag and drop the column to another place (almost all participants attempted to do this). Columns should have an option to manage its content (add, remove, edit, rename and reorder panels) and must allow the management of the tools connected to each column. Let people develop their own widgets for the dashboard (either elements in the columns or whole columns).

The observations also revealed that people were very actively exploring the interface, and learnt how to use it immediately or within one or two tries. This implies that the interface also supports fast learning, and motivates users to explore its functionality. Participants related many actions and interface elements to the tools or environments they are familiar with - Facebook, iOS, OS X, Gmail. It should be possible to collapse columns into tabs or icons, so that they can be opened easily and immediately when needed, but are not taking place on the screen when not needed (analogy to browser tabs, or minimizing applications in OS).

The participants highly valued the aggregation of the tools they usually use. As one designer explains: "I think I would really love it. Because from here, from this interface, I can easily go straight to certain file, certain person, (...) instead of searching: Where is this? Where is that?" [ppn1]. Another designer commented: "It's handy because you don't need to learn all the new tools. If someone prefers this and someone prefers others" [ppn3].

Since the dashboard integrates many activities, services and files, participants noted that there should be a visible confirmation after each action with the possibility to undo the action. Some suggested that, ideally, the dashboard should have a full integration with the connected services and with local files and that the interface should point directly to the aggregated content. Finally the interface should provide means for immediate interaction (instant message or email) with the people in the list of contacts.

\section{DISCUSSION}

Designers use many general-purpose tools for different collaborative tasks. Mastering the diversity and combination of the tools and functionalities, and keeping an overview of the process is a recurrent problem in design practice. The present study has explored means to link different tools, to filter relevant information across them and to switch easily between tasks during collaborative design projects. Through two design iterations followed by user tests, it was confirmed the relevance of a dashboard application presenting status information compiled from several general purpose tools for enabling designers to maintain an overview of their work and of the activities of the design team.

So far, feedback from users confirms the general design direction chosen: the dashboard should combine information from different but already familiar tools [7], rather than attempting to replace them. The first iteration identified the importance of optimizing the dashboard for visual scanning and support the search of content. The second iteration identified one more important requirement: to allow users to customize the dashboard as needed (by individual preference, role, workload, number of projects and team size).

Awareness is critical for collaboration. It provides an understanding of others and their activities and it helps guiding ones actions [2]. Awareness is dynamically built through practices [20]. In the design studio, the use of space and artifacts support the creation of awareness [23]. 
However, on remote environments, different awareness mechanisms should be developed to support seamless collaboration [4]. Through the integration and visualization of the different collaboration tools, Peepdeck aims to facilitate some the most relevant components of the design practice: social interactions supported by the exchange of artifacts [22] and information. Additionally, Peepdeck uses a user-centered approach [5] and adopts several awareness mechanisms such as personalization [4], workspace awareness [6], team availability [3] and work progress [2]. Further iterations involving different methods of evaluation on real scenarios must be done to ensure the generalization, precision and realism of these findings [14]. One can imagine that tools like Peepdeck can support other professionals for other types of collaborative work e.g., to quickly interact with the team (through sending emails or instant messages directly to team members), keeping and self-updated about the project development (following the activity of the colleagues, following the deadlines, tasks and their statuses), and accessing the shared resources easily.

\section{CONCLUSION}

Two iterations were presented of the design, prototyping and testing of PeepDeck an application that aggregates information from tools and online services that are popular amongst design teams, allowing them to be aware of project work. This process identifies several requirements for the design of tools to support collaboration awareness for design teams, these are: a) support the use of collections of widespread tools rather than replace them with a special purpose one b) design the system as a glanceable display to support awareness and peripheral interaction c) allow customization to individual needs and practices.

While these requirements have been identified in related literature regarding collaborative work, the emphasis on the combining general-purpose tools for supporting design activities is new. Further, the notion of personalization and glanceability refer to specific needs of design teams, which differ from the interpersonal awareness applications that have occupied CSCW literature in the past. Future work will explore how functional prototypes of such awareness functionality can be implemented and the extent to which they can be accepted by design teams and to which they succeed in fostering awareness.

\section{REFERENCES}

[1] S. Coradeschi and A. Saffiotti, "Perceptual anchoring of symbols for action", in: Proceedings of the 17th International Joint Conference on Artificial Intelligence (IJCAI), Seattle, WA, pp. 407-412, 2001

[2] P. Dourish and V. Bellotti, "Awareness and coordination in shared workspaces". In Proceedings of the ACM CSCW '92 Conference on Computer Supported Cooperative Work, pp. 107-113, 1992

[3] W. W. Gaver, "The Affordances of Media Spaces for Collaboration", In Proceedings of the Conference on Computer-Supported Cooperative Work - CSCW'92 (Oct. 31-Nov. 4, Toronto, Canada). N.Y.: ACM, pp. 17-24, 1992
[4] T. Gross, "Supporting Effortless Coordination: 25 Years of Awareness Research", Computer Supported Cooperative Work, vol. 22, pp. 425474, 2013

[5] T. Gross, C. Stary and A. Totter, "User-Centered Awareness in Computer-Supported Cooperative Work-Systems: Structured Embedding of Findings from Social Sciences", International Journal of Human-Computer Interaction (IJHCI), Vol. 18, no. 3, pp. 323-360, 2005

[6] C. Gutwin and S. Greenberg, "A Descriptive Framework of Workspace Awareness for Real- Time Groupware", Computer Supported Cooperative Work: The Journal of Collaborative Computing, Vol. 11, no. 3-4, pp.411-446, 2002

[7] P. Hekkert, D. Snelders, and P. C. W. Van Wieringen, “ 'Most advanced, yet acceptable': Typicality and novelty as joint predictors of aesthetic preference in industrial design". British Journal of Psychology, 94, 1, pp. 111-124, 2003

[8] J. Holmgren, J. Juola and R. Atkinson, "Response latency in visual search with redundancy in the visual display", Percept. Psychophys. 16, pp. 123-128, 1974

[9] J. Juola, Cognitive Psychology, Cengage Learning, 2009

[10] H. Karsten and M. Jones, "The long and winding road: Collaborative IT and organisational change" in Int. Conference on Computer Supported Work (CSCW'98), 1998

[11] T. Matthews, "Designing and evaluating glanceable peripheral displays" in Proceedings of the 6th conference on Designing Interactive systems, pp. 343-345, 2006

[12] J. E. McGrath, "Groups: Interaction and performance", Vol. 14. Englewood Cliffs, NJ: Prentice-Hall, 1984.

[13] J. Muñoz-Alcántara, P. Markopoulos and M. Funk, "Social Media as ad hoc Design Collaboration Tools" in Proceedings of the European Conference on Cognitive Ergonomics 2015 (ECCE '15), 2015

[14] D. C. Neale, J. M. Carroll and M. B. Rosson, "Evaluating computersupported cooperative work: models and frameworks" in Proceedings of the 2004 ACM conference on Computer supported cooperative work (CSCW '04), pp. 112-121, 2004

[15] D. Norman, The Design of Everyday Things, The MIT Press, London, 1998

[16] R. Oppermann, User Interface Design, Institute for Applied Information Technology, GMD Forschungszentrum Informationstechnik, Germany, 2002

[17] W. Orlikowski, "Sociomaterial Practices: Exploring Technology at Work," Organization Studies, 28, pp. 1435-1448, 2007

[18] V. Pipek and V. Wulf, "Infrastructuring: Towards an Integrated Perspective on the Design and Use of Information Technology", in Journal of the Association of Information System (JAIS), Vol. 10, Issue 5, pp. 306-332, 2015

[19] C. Rossitto, C. Bogdan and K.S. Eklundh, "Understanding Constellations of Technologies in Use in a Collaborative Nomadic Setting" in CSCW Journal, vol. 23, no. 2, 2014

[20] K. Schmidt, "The Problem With Awareness: Introductory Remarks on Awareness in CSCW"., Computer Supported Cooperative Work: The Journal of Collaborative Computing, Vol. 11, no.3-4, pp. 285-298, 2002

[21] K. Schmidt and L. Bannon, "Constructing CSCW: The First Quarter Century" in Computer Supported Cooperative Work, 22: pp. 345-372, 2013

[22] K. Schmidt and I. Wagner, "Coordinative artefacts in architectural practice" in Proc. of COOP 2002, pp. 257-274, 2002

[23] D. Vyas, G. van der Veer and A. Nijholt, "Creative practices in the design studio culture: collaboration and communication" in Cognition, Technology \& Work, 15, 4, pp. 415-443, 2013

[24] V. Wulf, "Evolving Cooperation when Introducing Groupware - A Self-Organization Perspective" in Cybernetics and Human Knowing, 6(2), pp. $55-75,1999$ 